\title{
Asparaginyl ligase-catalyzed one-step cell surface modification of red blood cells
}

Thibault J. Harmand ${ }^{\mathrm{a}, \# \text {, Novalia Pishesha }}$ a,\#, Fabian B. H. Rehmª, Weiyi Ma ${ }^{\mathrm{a}}$, William B. Pinney Yushu J Xie ${ }^{a} \&$ Hidde L. Ploegh ${ }^{\text {a }}$

a. Program in Cellular and Molecular Medicine, Boston Children's Hospital, Harvard Medical School, Boston, Massachussets 02115, United States

b. Australian Research Council Centre of Excellence for Innovations in Peptide and Protein Science, Institute for Molecular Bioscience, The University of Queensland, Brisbane, QLD 4072, Australia

\# Contributed equally to this work

*Corresponding author. Email: hidde.ploegh@childrens.harvard.edu 


\section{Contents:}

1. Experimental section

1.1 General procedure for peptide synthesis

1.2 Peptide and probe synthesis

1.3 Recombinant production of C247A-OaAEP1

1.4 Screening of OaAEP1 RBCs labelling conditions

1.5 Biological characterization of modified RBCs

1.6 Type 1 Diabetes (T1D) Model in NOD/SCID mice

1.7 Sortase reaction with VHH 05 and VHH A12

1.8 RBCs labelling with nanobodies

2. Supplementary references 


\section{Experimental section}

\subsection{General procedure for peptide synthesis}

All reagents were obtained from commercial sources and used as purchased. Unless noted otherwise, all reactions were carried out at room temperature. Peptides and probes were purified on a C18 column, Gemini, $5 \mu \mathrm{m}, 10 \times 250 \mathrm{~mm}$; Phenomenex column. LC-MS spectra were recorded on a Waters Xevo system equipped with HPLC-C8 column at a flow rate of $3.0 \mathrm{~mL} / \mathrm{min}$.

Peptides, probes and linker were synthesized following the standard solid phase peptide synthesis (SPPS) protocol below:

1. Coupling condition: Fmoc-AA-OH (ChemPep) (5 equiv) HATU (Chemlmpex Int) (4.8 equiv), $N, N$ - diisopropylethylamine (Chemlmpex Int) (DIPEA) (10 equiv), in DMF (MiliporeSigma). After premixing for $15 \mathrm{sec}$, the pre- activated solution was added and agitated for $1 \mathrm{~h}$.

2. Deprotection condition: $20 \%$ piperidine (MiliporeSigma) in DMF, 2 X 5 min.

3. Cleavage condition: TFA (MiliporeSigma) / TIPS (MiliporeSigma) / $\mathrm{H}_{2} \mathrm{O}(95 / 2.5 / 2.5)$, $2 \mathrm{~h}$.

4. Purification and characterization: Cleavage solution $(<10 \mathrm{~mL})$ was precipitated in prechilled Ether (VWR) (40 mL), centrifuged and the supernatant was removed. Peptides were dissolved in $\mathrm{H}_{2} \mathrm{O}, \mathrm{CH}_{3} \mathrm{CN}$ or DMSO and purified by RP-HPLC with: solvent $A, 0.1 \%$ TFA in $\mathrm{H}_{2} \mathrm{O}$; solvent $\mathrm{B}, 0.1 \%$ TFA in $\mathrm{CH}_{3} \mathrm{CN}$. Gradient: $5 \%$ to $90 \%$ in $15 \mathrm{~min}$. Fractions containing pure product were collected and lyophilized. The list of peptides synthesized, exact mass and observed mass are combined in the table below.

\begin{tabular}{|l|l|l|}
\hline Peptides & Exact Mass $[\mathbf{M + H}]^{+}(\mathbf{D a})$ & Observed $\left.[\mathbf{M + H}]^{+} \mathbf{( D a}\right)$ \\
\hline Biotin-LPETGG & 799.37 & 799.67 \\
\hline Biotin-RNGL & 685.35 & 685.48 \\
\hline Biotin-p31 & 2060.02 & 2059.84 \\
\hline Trivalent linker & 1050.50 & 1050.61 \\
\hline Linker-Alexa Fluor 488 & 1747.59 & 1747.58 \\
\hline Linker-Pacific Blue & 1456.60 & 1456.76 \\
\hline TAMRA-Ubc6e TAG & 2204.11 & 2204.51 \\
\hline
\end{tabular}

\subsection{Synthesis of the peptides, probes and linkers}




\section{Biotin-LPETGG (1)}

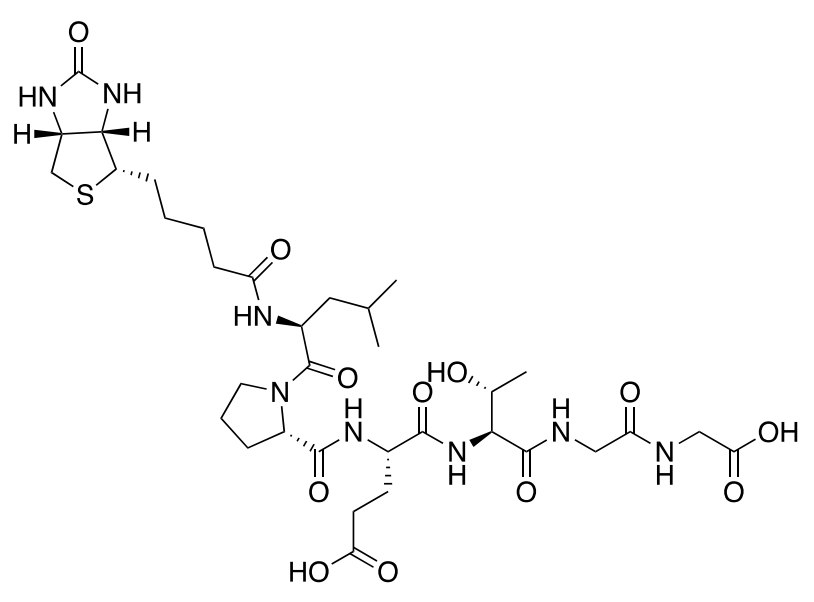

The peptide was assembled until the terminal leucine residue following the protocol previously described. After Fmoc deprotection, After Fmoc deprotection, biotin (MiliporeSigma) (5 equiv), HATU (4.8 equiv) were dissolved in $\mathrm{CH}_{2} \mathrm{Cl}_{2} / \mathrm{DMF} 1: 1$ and DIPEA (10 equiv) was added. The resulting mixture was activated for 1 min at r.t, added to the resin and agitated for 5 hours at r.t. The resin was then washed with DMF and $\mathrm{CH}_{2} \mathrm{Cl}_{2}$ several times, dried, cleaved and purified on RP-HPLC using the standard protocol. Exact mass for 1: $\mathrm{C}_{34} \mathrm{H}_{55} \mathrm{~N}_{8} \mathrm{O}_{12} \mathrm{~S}[\mathrm{M}+\mathrm{H}]^{+}$was 799.37 , found 799.67 .

\section{Biotin-RNGL (2)}

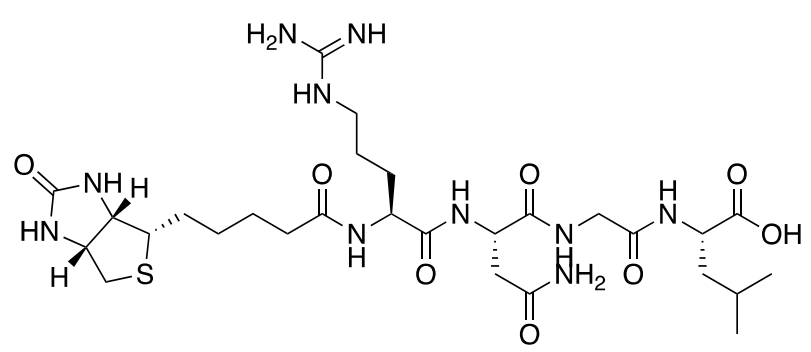

The peptide was assembled until the arginine residue following the protocol previously described. After Fmoc deprotection, biotin (MiliporeSigma) (5 equiv), HATU (4.8 equiv) were dissolved in $\mathrm{CH}_{2} \mathrm{Cl}_{2} / \mathrm{DMF}$ 1:1 and DIPEA (10 equiv) was added. The resulting mixture was activated for $1 \mathrm{~min}$ at r.t, added to the resin and agitated for 5 hours at r.t. The resin was then washed with DMF and $\mathrm{CH}_{2} \mathrm{Cl}_{2}$ several times, dried, cleaved and purified on RP-HPLC using the standard protocol. Exact mass for 2: $\mathrm{C}_{28} \mathrm{H}_{49} \mathrm{~N}_{10} \mathrm{O}_{8} \mathrm{~S}$ $[\mathrm{M}+\mathrm{H}]^{+}$was 685.35 , found 685.48 . 


\section{Biotin-p31 (3)}

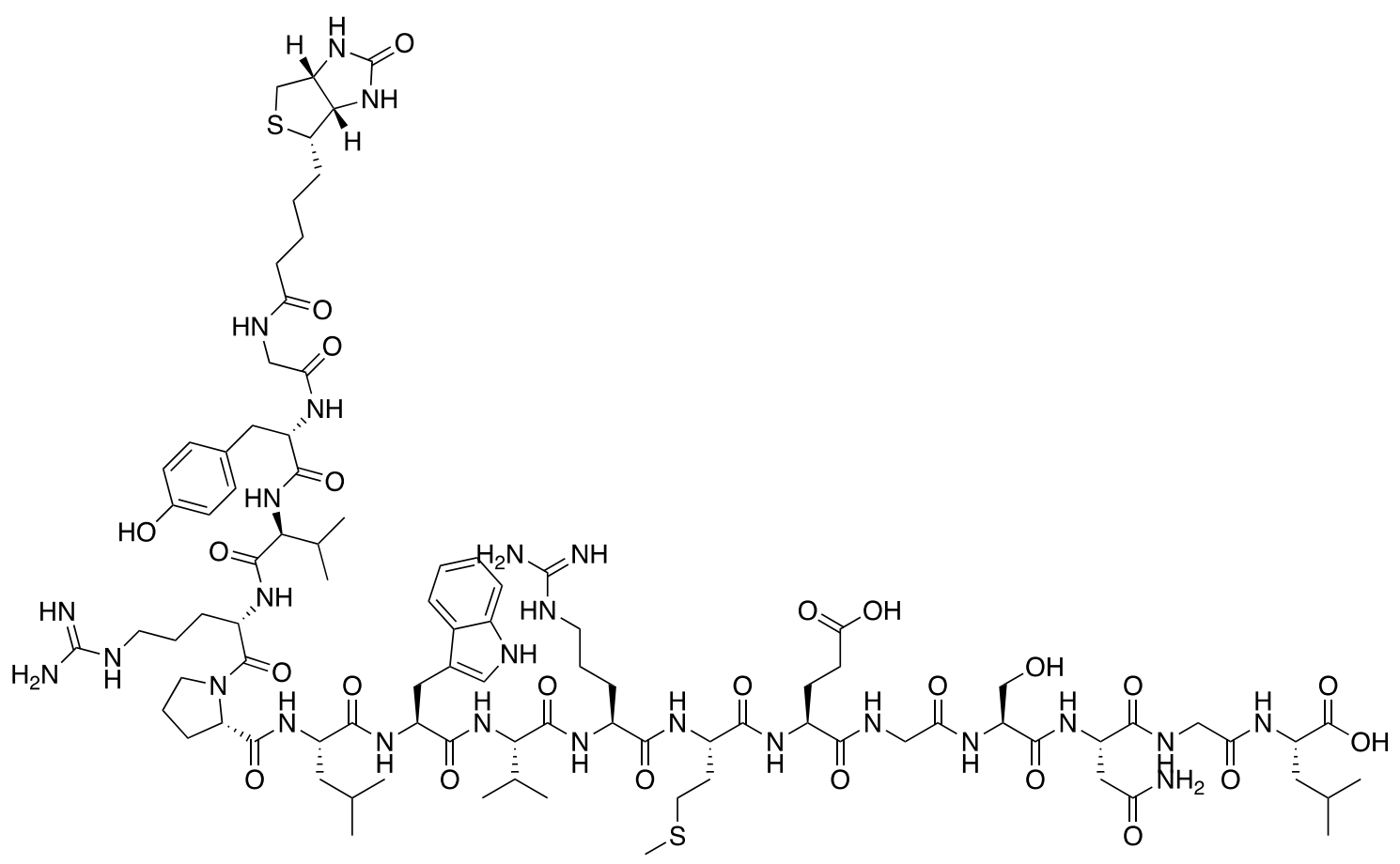

The peptide was assembled until the glycine residue following the protocol previously described. After Fmoc deprotection, biotin (MiliporeSigma) (5 equiv), HATU (4.8 equiv) were dissolved in $\mathrm{CH}_{2} \mathrm{Cl}_{2} / \mathrm{DMF}$ 1:1 and DIPEA (10 equiv) was added. The resulting mixture was activated for $1 \mathrm{~min}$ at r.t, added to the resin and agitated for 5 hours at r.t. The resin was then washed with DMF and $\mathrm{CH}_{2} \mathrm{Cl}_{2}$ several times, dried, cleaved and purified on RP-HPLC using the standard protocol. Exact mass for 3: $\mathrm{C}_{92} \mathrm{H}_{143} \mathrm{~N}_{26} \mathrm{O}_{24} \mathrm{~S}_{2}$ $[\mathrm{M}+\mathrm{H}]^{+}$was 2060.02, found 2059.84 .

\section{Trivalent linker (4)}

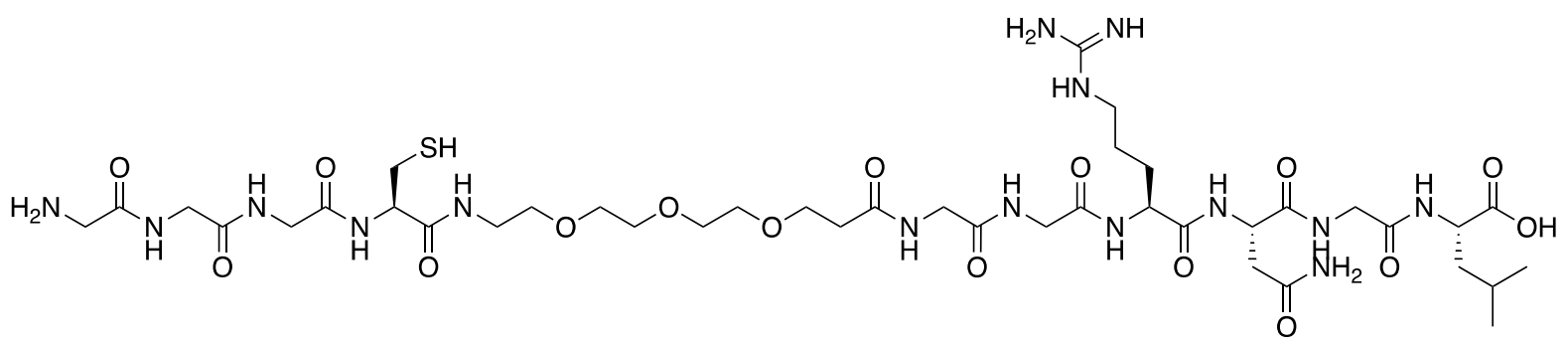

The peptide was assembled until the cysteine residue following the protocol previously described. After Fmoc deprotection, Boc-Gly-Gly-Gly-OH (Chemlmpex) (5 equiv) HATU (4.8 equiv) were dissolved in DMF and DIPEA (10 equiv) was added. The resulting mixture was activated for $1 \mathrm{~min}$ at r.t, added to the resin and agitated for 5 
hours at r.t. The resin was then washed with DMF several times, dried, cleaved and purified on RP-HPLC using the standard protocol. Exact mass for 4: $\mathrm{C}_{40} \mathrm{H}_{72} \mathrm{~N}_{15} \mathrm{O}_{16} \mathrm{~S}^{+}$ $[\mathrm{M}+\mathrm{H}]^{+}$was 1050.50 , found 1050.61

Linker-AlexaFluor 488 (5)

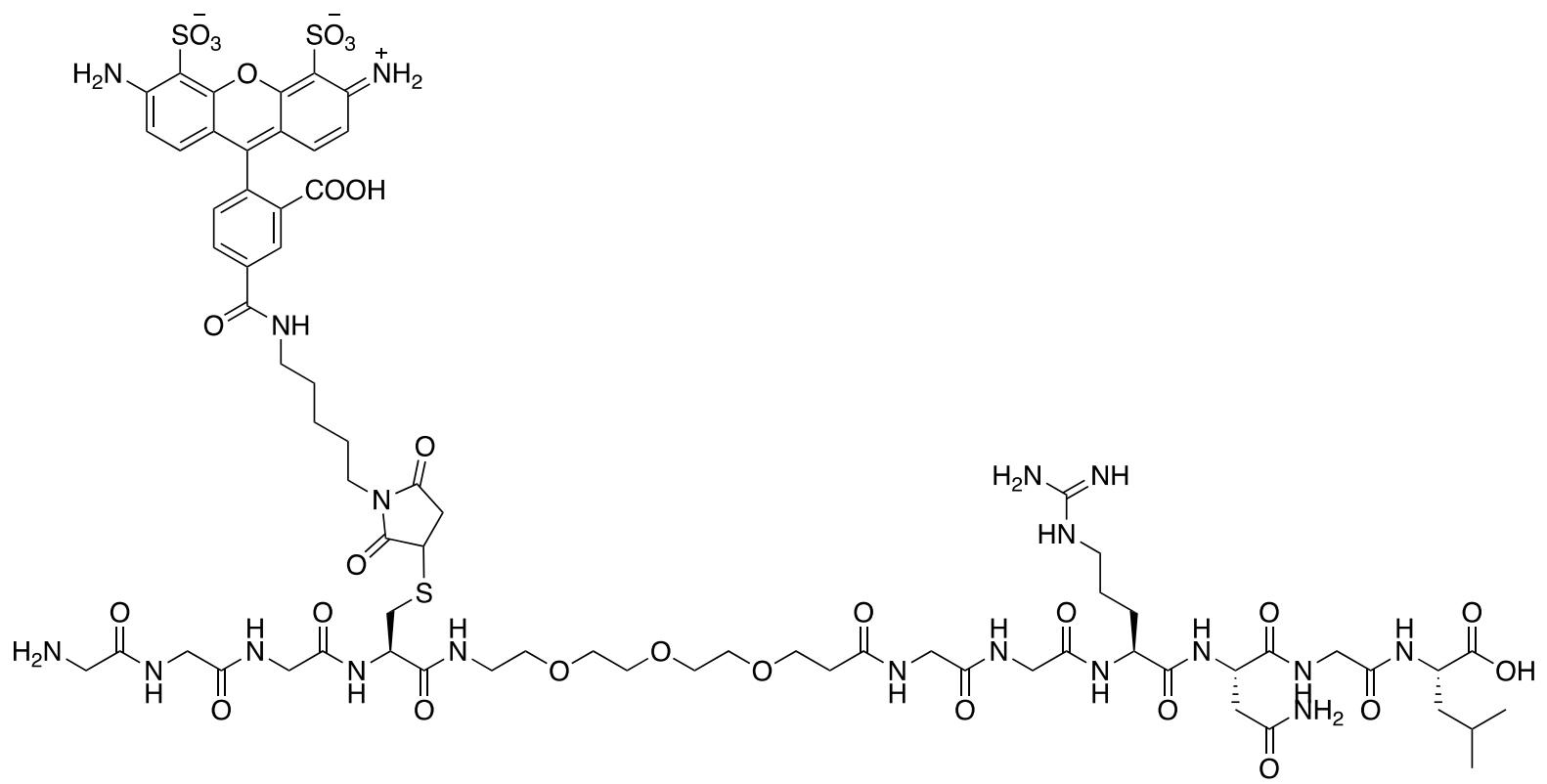

The trivalent linker (8 mg, $7.6 \mu \mathrm{mol})$ was dissolved in DMSO $(250 \mu \mathrm{L})$. AlexaFluor 488 maleimide (ThermoFisher) $(1 \mathrm{mg}, 1.4 \mu \mathrm{mol})$ was dissolved in DMSO $(150 \mu \mathrm{L})$ then added to peptide solution. After shaking at $\mathrm{r}$.t for $4 \mathrm{~h}$, the reaction mixture was directly purified by RP-HPLC, solvent $A, 0.1 \%$ TFA in $\mathrm{H}_{2} \mathrm{O}$; solvent $\mathrm{B}, 0.1 \%$ TFA in $\mathrm{CH}_{3} \mathrm{CN}$. Gradient: $5 \%$ to $90 \%$ in 15 min. Fractions containing pure product were collected and lyophilized. Exact mass for $5: \mathrm{C}_{70} \mathrm{H}_{97} \mathrm{~N}_{19} \mathrm{O}_{28} \mathrm{~S}_{3}[\mathrm{M}+\mathrm{H}]^{+}$was 1747.59 , found 1747.58

Linker-Pacific Blue (6) 
<smiles>CC(C)C[C@H](NC(=O)CNC(=O)[C@H](CC(=O)N[C@@H](CCCCNC(=N)N)C(=O)N[C@@H](CC(=O)NCC(=O)NCC(=O)CCOCCOCCOCCNC(=O)[C@H](CSC1CC(=O)N(CCCCCCNC(=O)c2cc3cc(F)c(O)c(F)c3oc2=O)C1=O)NC(=O)CNC(=O)CNC(=O)CN)C(=O)O)C(=O)O)C(=O)O</smiles>

The trivalent linker (8 mg, $7.6 \mu \mathrm{mol})$ was dissolved in DMSO $(250 \mu \mathrm{L})$. Pacific Blue maleimide (ThermoFisher) (1 mg, $2.5 \mu \mathrm{mol})$ was dissolved in DMSO (150 $\mu \mathrm{L})$ then added to peptide solution. After shaking at r.t for $4 \mathrm{~h}$, the reaction mixture was directly purified by RP-HPLC, solvent $A, 0.1 \%$ TFA in $\mathrm{H}_{2} \mathrm{O}$; solvent $\mathrm{B}, 0.1 \%$ TFA in $\mathrm{CH}_{3} \mathrm{CN}$. Gradient: $5 \%$ to $90 \%$ in $15 \mathrm{~min}$. Fractions containing pure product were collected and lyophilized. Exact mass for 6: $\mathrm{C}_{59} \mathrm{H}_{88} \mathrm{~F}_{2} \mathrm{~N}_{17} \mathrm{O}_{22} \mathrm{~S}^{+}[\mathrm{M}+\mathrm{H}]^{+}$was 1456.60, found 1456.76

\section{TAMRA-Ubc6e TAG (7)}

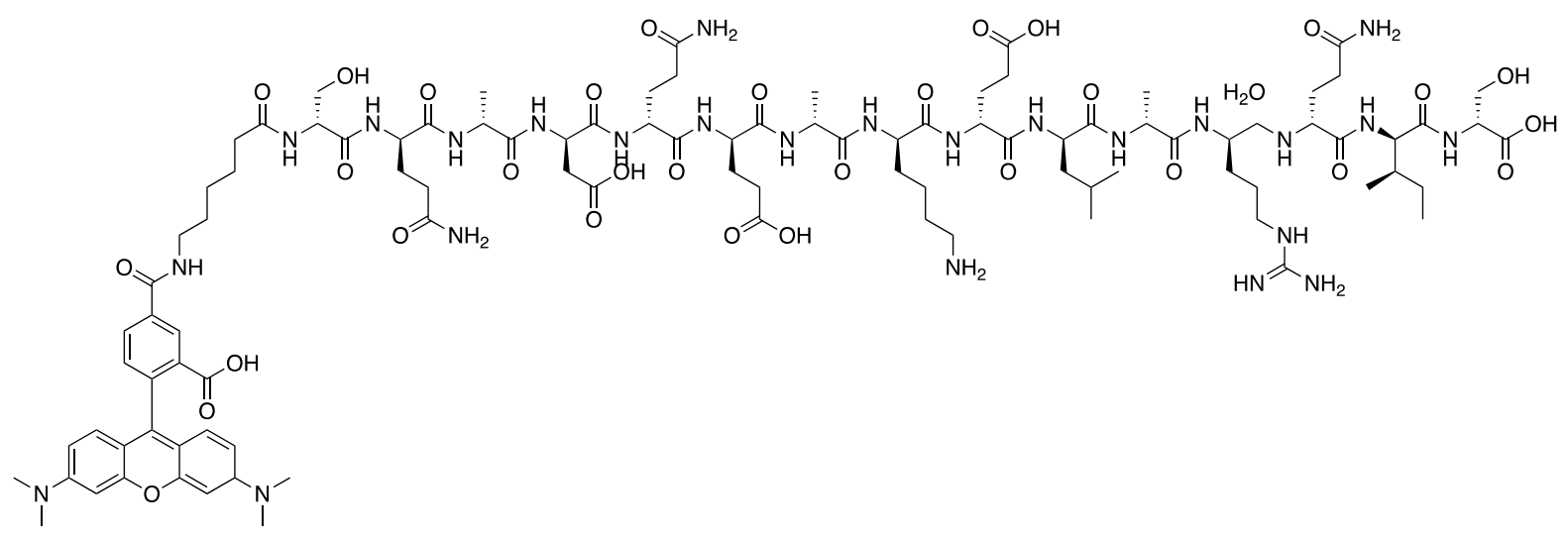

The peptide was assembled until the arginine residue following the protocol previously described. After Fmoc deprotection, 5-TAMRA (ThermoFisher) (3 equiv), HATU (2.5 equiv) were dissolved in DMF and DIPEA (6 equiv) was added. The resulting mixture was activated for $1 \mathrm{~min}$ at r.t, added to the resin and agitated for 5 hours at r.t. The resin was then washed with DMF several times, dried, cleaved and purified on RPHPLC using the standard protocol. Exact mass for 7: $\mathrm{C}_{99} \mathrm{H}_{153} \mathrm{~N}_{25} \mathrm{O}_{32}[\mathrm{M}+\mathrm{H}]^{+}$was 2204.11, found 2204.51. 


\subsection{Recombinant production of C247A-OaAEP1}

C247A-OaAEP1 was produced in E. coli SHuffle as previously described ${ }^{1-3}$. Briefly, 6-His-Ubiquitin-OaAEP (C247A) inserted in pHUE and transformed into E. coli SHuffle was expressed overnight at $18^{\circ} \mathrm{C}$. Clarified lysates were then purified by NiNTA and SEC. Auto-activation of the AEPs was then induced by titrating acetic acid into the solution to reach $\mathrm{pH} 4-4.5$ and incubated at $37^{\circ} \mathrm{C}$ for $5-8$ hours as monitored by band shifts on SDS-PAGE. The mature enzyme was then purified further by cation exchange (HiTrap SP FastFlow) and eluted with $50 \mathrm{mM}$ sodium acetate buffer containing $10 \%$ glycerol, $1 \mathrm{M} \mathrm{NaCl}$, at $\mathrm{pH}$ 4.0. Pure enzyme was then quantified by $\mathrm{A}_{280}$ and stored in aliquots at $-80^{\circ} \mathrm{C}$ until use.

\subsection{Screening of OaAEP1 RBCs labelling conditions}

\subsubsection{Mice}

All animals were housed in the animal facility of Boston Children's Hospital $(\mathrm{BCH})$ and were maintained according to protocols approved by the $\mathrm{BCH}$ Committee on Animal Care. C57BL/6J mice, which is the source of RBCs used in all of the subsequent experiments, were either purchased from the Jackson Laboratory or bred in house.

\subsubsection{Screening of labeling conditions}

Note: All experiments were done with $1 \mathrm{mM}$ biotin-RNGL

In an Eppendorf tube, 20 millions of RBCs were incubated with biotin-RNGL peptide in $20 \mu \mathrm{L}$ of PBS and different concentration of OaAEP1 was added. At the different time point, $1 \mathrm{~mL}$ of PBS was added to the Eppendorf tubes, transferred to $8 \mathrm{~mL}$ tubes and centrifuged down at 1500rpm for $5 \mathrm{~min}$. The supernatant was discarded, $5 \mathrm{~mL}$ of FACS buffer (2 mM EDTA and 1\% FBS in PBS) was added and the cells were centrifuged down again at the same speed. This step was repeated 2 times to make sure that no enzyme and no biotin probe were left. After the final spin, the cells were re suspended in $300 \mu \mathrm{L}$ of FACS buffer and analyzed (Supplementary Figure 1 and 2). 


\section{OaAEP1}

concentration
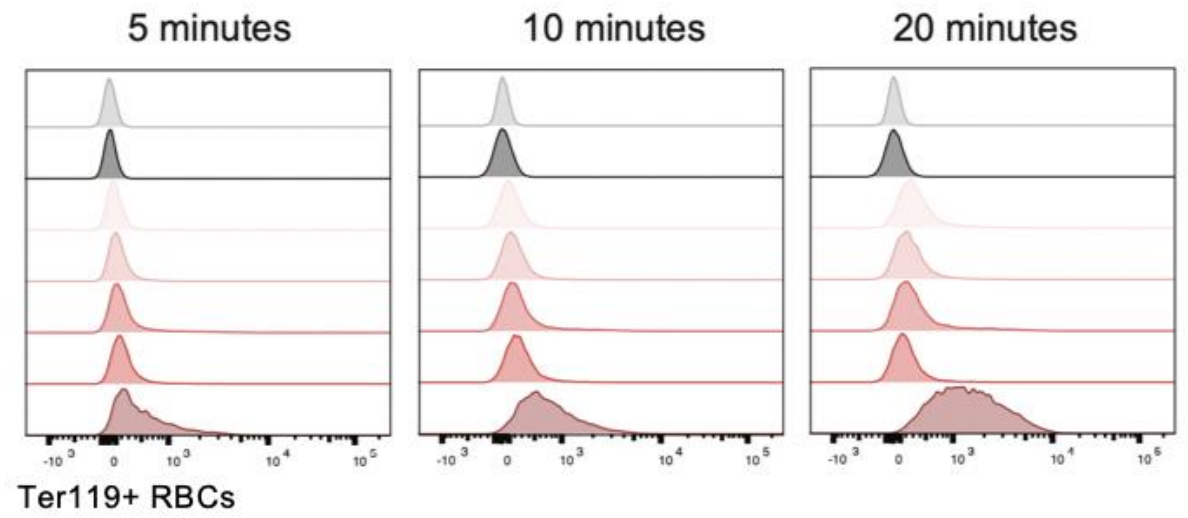

$2 \mu \mathrm{M}$

Ter119+ RBCs

\section{OaAEP1}

concentration
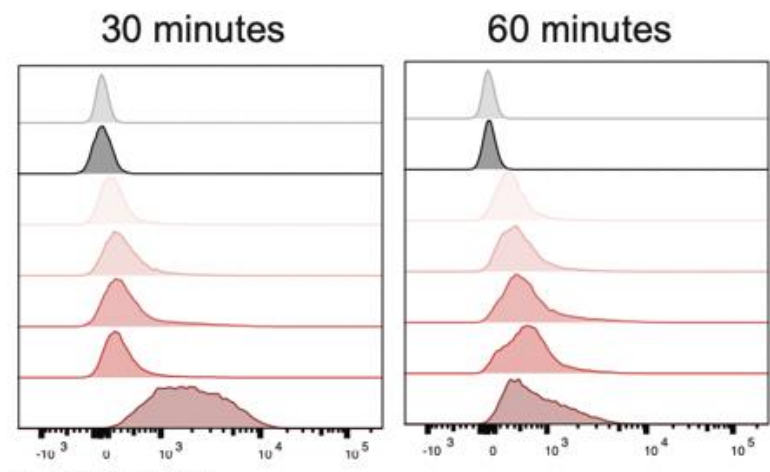

Ter119+ RBCs

Supplementary Figure 1: RBCs labeling using OaAEP1; enzyme concentration and reaction time monitoring by flow cytometry

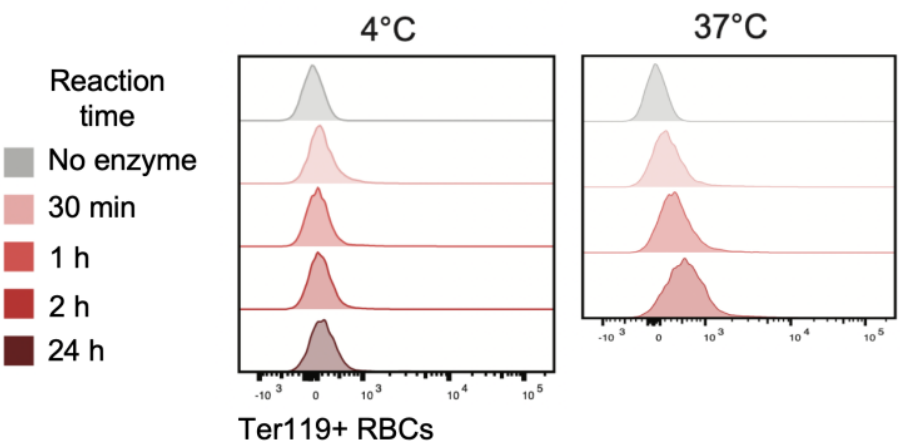

Supplementary Figure 2: RBCs labeling using OaAEP1; temperature and reaction time monitoring by flow cytometry

\subsubsection{Comparison with sortase}


In an Eppendorf tube, 100 million of RBCs were incubated with $1 \mathrm{mM}$ of biotin-RNGL and $50 \mathrm{nM}$ of OaAEP1 at $37^{\circ} \mathrm{C}$ for 2 hours. The cells were washed 3 times with PBS to make sure that no enzyme and no biotin probe were left. In parallel, and similarly, 100 million of RBCs were incubated with $1 \mathrm{mM}$ of biotin-LPETGG and $50 \mu \mathrm{M}$ of sortase A at $37^{\circ} \mathrm{C}$ for 2 hours. The cells were washed 3 times with PBS to make sure that no enzyme and no biotin probe were left.

After the washing steps, the labeling efficiency of these two enzymes was analyzed by SDS-PAGE and Western Blot. 8 million of sortase A- and OaAEP1-modified RBCs were loaded in each lane and analyzed by Coomassie staining and Western Plot using HRP-conjugated streptavidin. Coomassie staining insure that the number of cells loaded in each lane is the same.

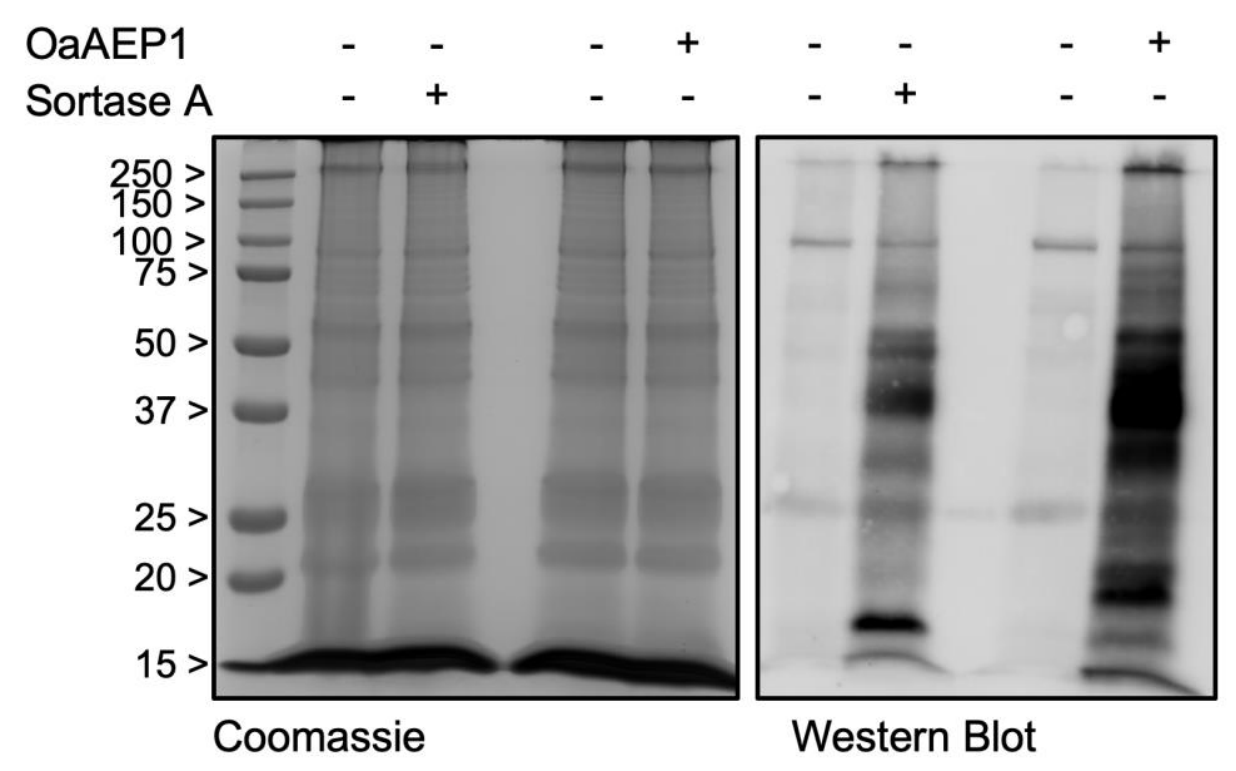

Supplementary Figure 3: Coomassie and Western blot analysis of RBCs labeling with biotinylated peptide using sortase A and OaAEP1.

\subsubsection{Estimation of amount of protein labelled with OaAEP1}

RBCs were labeled as described above and 8 millions of unlabeled and biotin-labeled RBCs were loaded per lane. To have an estimate of the amount of RBC proteins modified by OaAEP1 and Sortase were loaded per well. We then carried out western blot assay using Streptavidin-HRP (ThermoFisher Scientific, N100) at 1:10,000 
dilution. The copy number of recombinant human GPA protein per erythroid cell was derived using a linear signal intensity plot $\left(r^{2}=0.92\right)$ generated with 5,10 , and $20 \mathrm{ng}$ of biotinylated-GFP. As determined by the biotin signal intensity in the western blot with a Streptavidin-HRP and as quantified by Image Studio Lite, 8 millions RBCs contained $437 \mathrm{ng}$ and $254 \mathrm{ng}$ biotinylated proteins upon modification with OaAEP1 and Sortase, respectively.

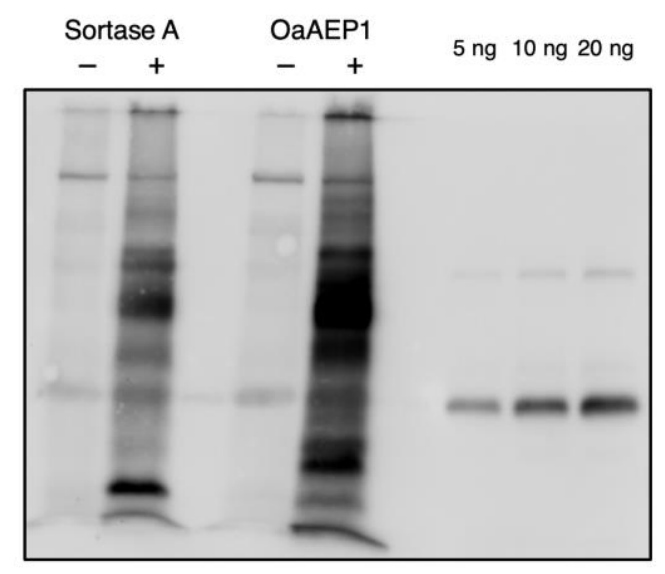

Supplementary Figure 4: Modified protein on RBCs quantification by Western blot and comparison between Sortase A and OaAEP1.

\subsection{Biological characterization of modified RBCs}

\subsubsection{Morphology assay}

Upon modification, RBCs were centrifuged onto poly-L-lysine-coated slides for $5 \mathrm{~min}$ at 400rpm (Thermo Shandon, Cytospin 3). RBCs were air-dried, fixed in cold methanol for $15 \mathrm{~min}$ at room temperature, and stained with 3,3'-Diaminobenzidine (DAB) according to the manufacturer's protocol (Sigma). Stained sections were imaged using a Keyence BZ-X800, magnification 20x.

\subsubsection{Live and death cell assay}

Upon modification, RBCs were stained with Annexin V and 7-AAD Apoptosis kit (ThermoFisher Scientific, A35136) as per the manufacturer's protocol prior to flow cytometry analyses. 


\subsubsection{In vivo circulatory lifespan assay}

Upon modification, RBCs were cytoplasmically labelled with Far-red CellTrace (ThermoFisher Scientific, C34571) as per the manufacturer's protocol prior to transfusion into recipient C57BL6/J mice. At the indicated time points, $20 \mu \mathrm{l}$ of blood were collected from these mice and washed twice and resuspended in PBS. We then carried out flow cytometry analyses on these RBCs, i.e. far-red fluorophore channel, to measure the fraction of the transfused RBCs remaining in the circulation.

\subsection{Type 1 Diabetes (T1D) Model in NOD/SCID mice}

\subsubsection{T1D induction}

NOD/SCID and NOD/BDC2.5 mice were either purchased from the Jackson Laboratory or bred in house.

Spleen and inguinal lymph nodes were harvested from 7-9-week-old BDC2.5 mice. Cells were resuspended in complete RPMI (RPMI supplemented with 2mM glutaMAX, $10 \mathrm{mM}$ HEPES, Non-Essential Amino Acids, $1 \mathrm{mM}$ Sodium pyruvate, 55uM 2mercaptoethanol, 10\% heat-inactivated FBS) supplemented with $0.5 \mu \mathrm{M}$ p31 peptide (BDC2.5 mimotope, GenScript) and plated in tissue culture dishes at 1 million cells $/ \mathrm{mL}$. After four days, cells were harvested, washed twice and resuspended in PBS. 5 million cells were adoptively transferred into 9-12-week-old female NOD.SCID mice via retro-orbital injection. $200 \mu$ l of RBCs or RBC-p31 were infused into the mice a day later as indicated. Blood glucose measurements were carried out every other day for 2 weeks and weekly for up to 1-2 months. Mice were considered diabetic when their blood glucose level exceeded $260 \mathrm{mg} / \mathrm{dL}$ for two subsequent weeks as measured by using the Active meter (Accu-Chek) (range 20-600 mg/dL) with corresponding Aviva Plus test strips (Accu-Check).

Mice were sacrificed via asphyxiation at the 2-month endpoint or when blood glucose levels exceeded $600 \mathrm{mg} / \mathrm{dL}$ for two subsequent weeks. The pancreas was fixed for further immunohistochemistry analysis, i.e. H\&E staining (Harvard Medical School Rodent Histology Core Facility). We also collected spleens, inguinal/pancreatic lymph nodes and pancreas were harvested and subjected for flow cytometry analysis at the endpoint. 


\subsubsection{Flow Cytometry Analyses}

Cells were harvested from spleen, lymph nodes, or other organs and were dispersed into RPMI1640 through a 40-micron cell strainer using the back of a $1 \mathrm{~mL}$ syringe plunger. Cell mixture were subjected to hypotonic lysis $\left(\mathrm{NH}_{4} \mathrm{Cl}\right)$ to remove red blood cells, washed twice in FACS buffer (2 mM EDTA and 1\% FBS in PBS) and resuspended in FACS buffer containing the corresponding fluorescent dyeconjugated antibodies. All staining was carried out at 1:100 dilution and with Fc block for 30 min at $4^{\circ} \mathrm{C}$ in dark. Samples were washed twice with FACS buffer before further analysis. All flow data were acquired on a FACS Fortessa flow cytometer (BD Biosciences) and analyzed using FlowJo software (Tree Star).

The following is the list of antibodies used in this study:

\begin{tabular}{|l|l|l|l|r|}
\hline Target & Color & Clone & Manufacturer & Cat. Number \\
\hline CD19 & PE & 6 D5 & Biolegend & 115508 \\
\hline CD3 & BV421 & 17 A2 & Biolegend & 100228 \\
\hline CD4 & FITC & GK1.5 & Biolegend & 100406 \\
\hline CD45.1 & PeCy7 & A20 & eBioscience & $25-0453-82$ \\
\hline CD8 & APC-Cy7 & $53-6.7$ & BioLegend & 100714 \\
\hline $\begin{array}{l}\text { Fc block } \\
\text { (CD16/CD32) }\end{array}$ & N.A. & 93 & Biolegend & 101302 \\
\hline
\end{tabular}

\subsection{Sortase reaction with VHH 05 and VHH A12}

Sortase ligation were performed using sortase A heptamutant. $250 \mu \mathrm{M}$ of $\mathrm{VHH}$ was incubated with $1 \mathrm{mM}$ of the linker bearing the desired fluorescent dye and $30 \mu \mathrm{M}$ of Sortase A in PBS, overnight at $4{ }^{\circ} \mathrm{C}$. The reaction mixture was then filtered through NiNTA to remove the sortase and the cleaved GGHHHHHH and passed through a PD10 column to remove the excess of linker. The modified VHH was concentrated to a final concentration of $350 \mu \mathrm{M}$, analyzed by LCMS and stored a $-80 \stackrel{\circ}{\circ}$

\subsubsection{MS traces of modified VHH 05 and VHH A12}

VHH05-Alexa488-NGL 


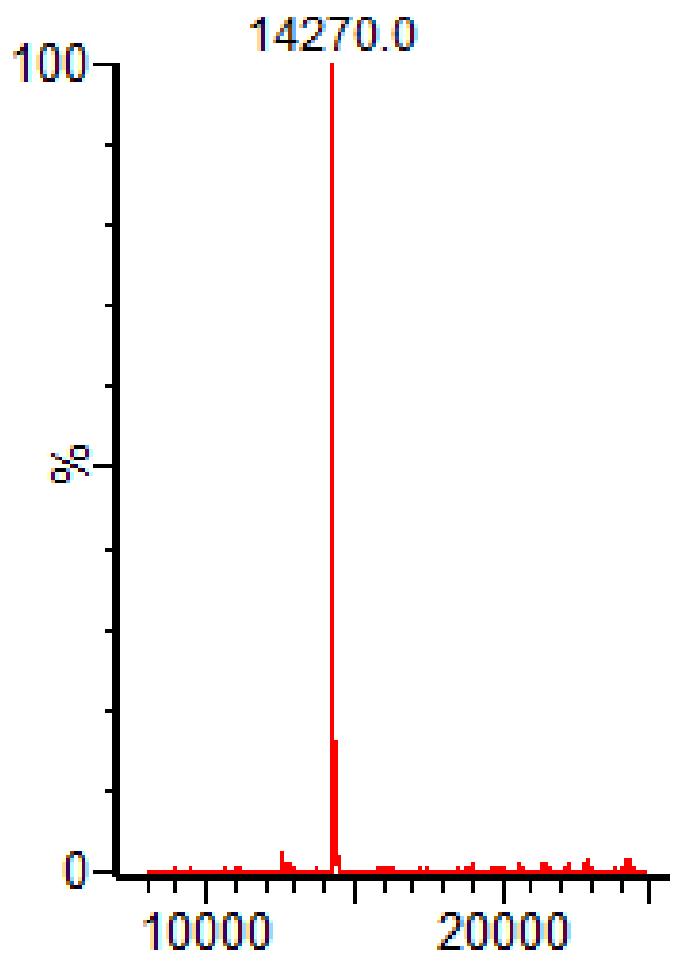

VHH A12-PacificBlue-NGL

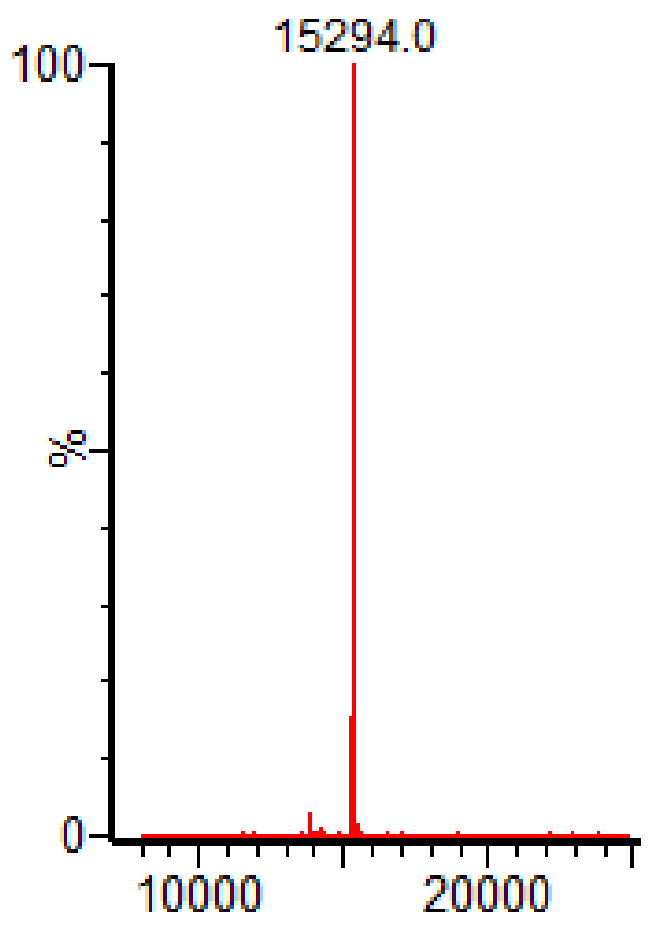

1.8 RBCs labelling with nanobodies

1.8.1 Mono labelling 
In an Eppendorf tube, 20 millions of RBCs were incubated with VHH-NGL in PBS at a final concentration of $200 \mu \mathrm{M}$ and final volume of $20 \mu \mathrm{L}$. $50 \mathrm{nM}$ of OaAEP1 was added and the reaction was gently mixed for 2 hours at $37^{\circ} \mathrm{C} .1 \mathrm{~mL}$ of PBS was added to the Eppendorf tubes to stop the reaction, transferred to $15-\mathrm{mL}$ conical tubes and centrifuged down at 1500rpm for $5 \mathrm{~min}$. The supernatant was discarded, $5 \mathrm{~mL}$ of FACS buffer (2 mM EDTA and 1\% FBS in PBS) was added and the cells were centrifuged down again at the same speed. This step was repeated 3 times to make sure that no enzyme and no unreacted $\mathrm{VHH}$ was left. After the last centrifugation, the cells were re-suspended in $300 \mu \mathrm{L}$ of FACS buffer and analyzed.

\subsubsection{Dual labelling}

The two VHHs were premixed in a 1:1 ratio and a final concentration for both of them of $150 \mu \mathrm{M}$. In an Eppendorf tube, 20 millions of RBCs were incubated with the 1:1 VHH mixture in PBS at a final concentration of $125 \mu \mathrm{M}$ and final volume of $20 \mu \mathrm{L}$. 50 $\mathrm{nM}$ of OaAEP1 was added and the reaction was gently mixed for 2 hours at $37^{\circ} \mathrm{C}$. The rest of the protocol is the same as previously described.

\subsubsection{Ubc6e tag B16 cell line}

B16 PDL1 KO cells containing a Ubc6e tag were generated by first knocking out PDL1 from WT B16F10 cells using CRISPR Cas. Single cell clonal populations were generated, and PDL1 KO was confirmed by sequencing. B16 PDL1 KO cells were then transduced with a lentivirus containing a transgene coding for surface displayed GFP linked to the Ubc6e tag, and FACS-sorted to generate the B16 PDL1 KO Ubc6e cell line

\subsubsection{Cancer cell line culture and preparation}

A20 and B16-Ubc6e tag were cultured in complete RPMI (RPMI supplemented with 2mM glutaMAX, 10 mM HEPES, Non-Essential Amino Acids, 1 mM Sodium pyruvate, $55 \mu \mathrm{M}$ 2-mercaptoethanol, 10\% heat-inactivated FBS). B16-Ubc6e tag cells were also GFP+. A20 cells were cytoplasmically labelled with Far-red CellTrace (ThermoFisher 
Scientific, C34571) as per the manufacturer's protocol prior to co-incubation with modified RBCs. 


\section{Supplementary references}

1. Jackson, M. A.; Gilding, E. K.; Shafee, T.; Harris, K. S.; Kaas, Q.; Poon, S.;

Yap, K.; Jia, H.; Guarino, R.; Chan, L. Y.; Durek, T.; Anderson, M. A.; Craik, D. J., Molecular basis for the production of cyclic peptides by plant asparaginyl endopeptidases. Nat Commun 2018, 9 (1), 2411.

2. Yang, R.; Wong, Y. H.; Nguyen, G. K. T.; Tam, J. P.; Lescar, J.; Wu, B., Engineering a Catalytically Efficient Recombinant Protein Ligase. J Am Chem Soc 2017, 139 (15), 5351-5358.

3. Harris, K. S.; Durek, T.; Kaas, Q.; Poth, A. G.; Gilding, E. K.; Conlan, B. F.; Saska, I.; Daly, N. L.; van der Weerden, N. L.; Craik, D. J.; Anderson, M. A., Efficient backbone cyclization of linear peptides by a recombinant asparaginyl endopeptidase. Nat Commun 2015, 6, 10199. 